\title{
$\backsim$ La normalización del Sistema de Información Ш del Patrimonio Histórico de Andalucía: El Tesauro de Patrimonio Histórico
}

\author{
Valle Muñoz Cruz \\ Centro de Documentación IAPH \\ Universidad de Sevilla
}

\section{El Sistema de Información del Patrimonio Histórico de Andalucía}

El objetivo de esta exposición es la presentación del proyecto de elaboración del Tesauro de Patrimonio Histórico, desarrollado por el Centro de Documentación del Instituto Andaluz del Patrimonio Histórico (IAPH) en colaboración con la Universidad y las Instituciones de Patrimonio; sin embargo se hace necesario contextualizarlo en el proceso de normalización del Sistema de Información del Patrimonio Histórico de Andalucía (SIPHA).
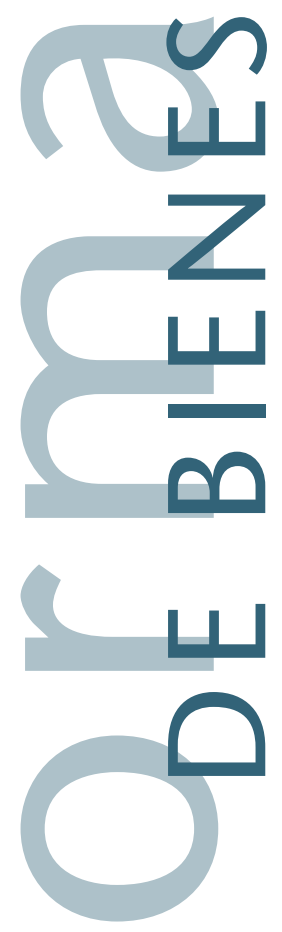

El SIPHA es el instrumento que se está gestionando para la sistematización de la información sobre los Bienes Culturales. El Tesauro de Patrimonio Histórico es el lenguaje documental que va a facilitar el tratamiento de la información y su eficaz recuperación por parte de todos los usuarios del Sistema.

La normalización ha sido un elemento importante, presente desde la puesta en marcha del Sistema de Información. En la actual fase de desarrollo del Sistema se procede a la integración de las diferentes bases de datos que lo configuran y hace aún más necesario el esfuerzo de normalización de todos los procesos documentales.

Es objetivo de esta exposición concienciar a todos los agentes de Patrimonio Histórico, que de alguna manera están implicados en la sistematización de la información, sobre la importancia de la normalización de todos los procesos realizados en el marco del Sistema de Información, y sobre la necesidad de su colaboración en esta labor.

Para comprender las actuaciones que sobre sistematización de la información de Patrimonio Histórico el Centro de Documentación viene desarrollando desde hace algunos años, en colaboración con la Universidad, la Dirección General de Bienes Culturales (DGBC) y las Instituciones del Patrimonio, es importante situarlas en el contexto general de la política de Patrimonio.

En este sentido se ha de destacar la importancia que el conocimiento del Patrimonio Histórico por parte de la Administración y de la sociedad en general tiene para la tutela del mismo, aspecto recogido en la ley de Patrimonio Histórico Español!. Así pues, es responsabilidad de la Administración instrumentar medidas que favorezcan el acceso a la información como factor fundamental para una política eficaz de los Bienes Culturales.
El Plan General de Bienes Culturales ${ }^{2}$ y el Decreto de Creación del $\mathrm{IAPH}^{3}$, siguiendo esta misma línea, establecen como uno de sus objetivos fundamentales "el conocimiento del Patrimonio Histórico mediante su documentación", porque difícilmente podrán tutelarse los Bienes Culturales sin un conocimiento exhaustivo de ellos.

Desde la importancia que el conocimiento y el acceso, a la información de los Bienes Culturales tiene para una tutela integral y efectiva del Patrimonio Histórico, en sus diferentes vertientes: gestión, investigación, difusión, etc. debe entenderse la configuración del Sistema de Información del Patrimonio Histórico de Andalucía.

El concepto de Sistema de Información está estrechamente relacionado con el de Gestión de la Información; ambos conceptos han alcanzado una importancia fundamental por cuanto implican organización y sistematización de los procesos documentales y de información de las organizaciones con el objetivo de favorecer la actividad que éstas desarrollan.

Esta aplicación de la Ciencia Documental, como medio para mejorar la actividad de cualquier entidad, implica la organización y sistematización de todos los flujos informativos tanto internos como externos de la misma. Sin duda en el fondo de esta aplicación de la Documentación ha de ubicarse "el paso de la concepción de información como servicio a la de recurso económico, a la de bien informativo"4. Efectivamente la información es un bien capaz de generar un valor determinado en cuanto que puede favorecer la gestión y el desarrollo de cualquier organización incidiendo en una acertada toma de decisiones.

La configuración y puesta en marcha de un Sistema de Información de Patrimonio Histórico ha de concebirse como una respuesta a las necesidades detectadas en materia de documentación e información sobre el Patrimonio Histórico, necesidades por otro lado constatadas en el Plan General de Bienes Culturales que recoge la necesidad de crear un Centro de Documentación que cubriera todas las deficiencias en esa materia. Un Centro de Documentación "que se nutriese de la información elaborada en los distintos campos de la política de los bienes culturales en Andalucía. La información almacenada recibiría controles temporales y sistemáticos a través de rutinas de trabajo y de campañas específicas de revisión. Este Centro de Documentación que podría ubicarse en el IAPH, estaría informatizado y prestaría información a los diversos agentes que intervienen en la tutela del Patrimonio Histórico, en su sentido más amplio y participativo, recibiendo a la vez de estos agentes otros datos actualizados o novedosos"5. Información que estaría a disposición de la Administración, investigadores y estudiosos del Patrimonio Histórico Andaluz, así como de entidades particulares y público en general. 


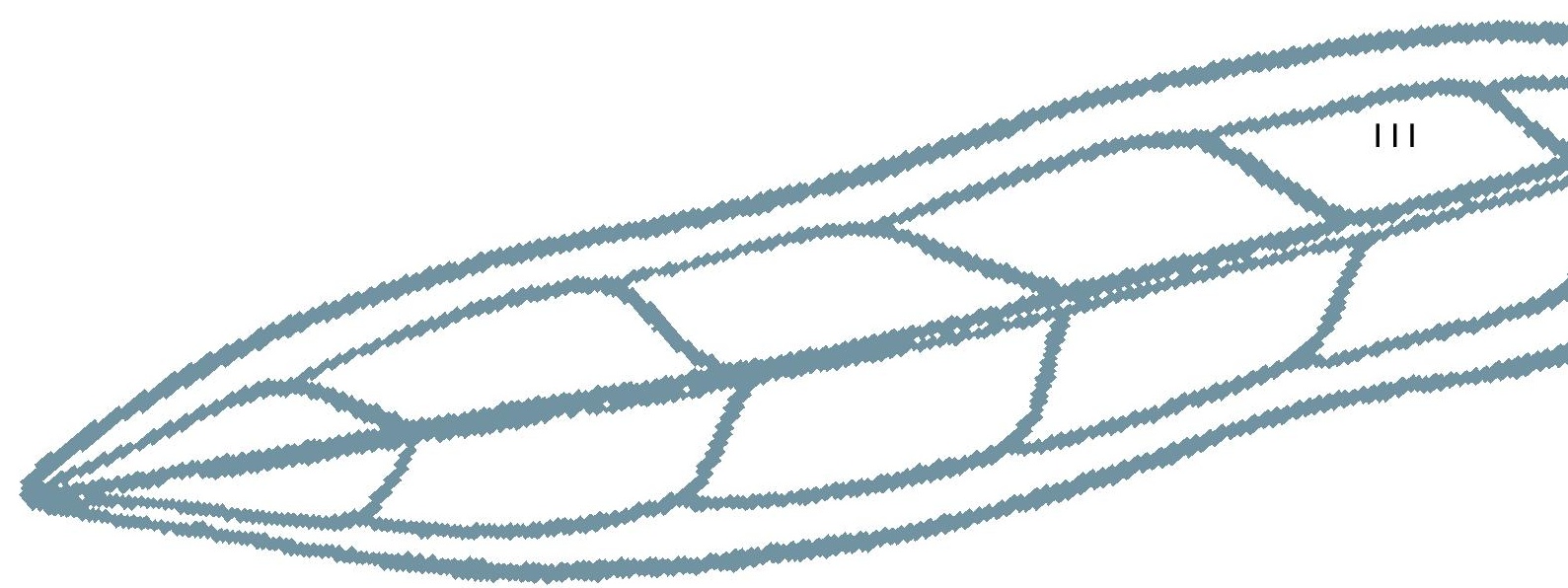

Efectivamente, como recoge el Plan General, los instrumenos de conocimiento que se habían utilizado hasta entonces (inventarios y catálogos) eran generalizadores e incompletos por cuanto ofrecían una visión de los Bienes Culturales descontextualizada, sin las implicaciones espaciales y ambientales que lo condicionan. Por otro lado, la aplicación de la documentación gráfica era escasa y la de las nuevas tecnologías inexistente. Era evidente la necesidad de racionalizar y sistematizar la documentación e información sobre los Bienes Culturales en el marco de un Sistema de Información, concebido como una estructura de información que posibilite el desarrollo de diferentes bases de datos y permita la interrelación del objeto con el territorio y el ambiente, mediante la aplicación de las Nuevas Tecnologías y de un soporte informático que facilite la integración de todas ellas y el acceso a esa información a través de una red informáti$\mathrm{ca}^{6}$. El SIPHA no sólo ofrece información sobre el Patrimonio Histórico sino que debe ser alimentado por los usuarios reales y potenciales del Sistema: Dirección General de Bienes Culturales (DGBC), Delegaciones Provinciales de Cultura (DDPP), IAPH, Instituciones de Patrimonio, Universidad...

El SIPHA permite la sistematización de toda la información generada y que se genere sobre los Bienes Culturales. Por tanto el desarrollo de este Sistema debe basarse en principios de integración y coordinación entre las instituciones y organismos que contienen documentación e información sobre Patrimonio Histórico. Son necesarios, por tanto, dos elementos fundamentales para el desarrollo del Sistema: coordinación y normalización. Sobre este último vamos a centrar nuestra exposición.

La puesta en marcha del SIPHA debe hacerse de forma modular y progresiva a través de la coordinación de todos aquellos proyectos que de alguna manera están sistematizando la documentación del Patrimonio Histórico. Fue así como en 1993 se puso en marcha un Programa de Coordinación de diferentes proyectos con objeto de hacer confluir los esfuerzos que se estaban produciendo en la misma dirección para la configuración del SIPHA. Algunos de estos proyectos son desarrollados directamente por el Centro de Documentación, otros en colaboración con la Universidad o los Museos y otros por la DGBC.

\section{La normalización del Sistema de Información del Patrimonio Histórico de Andalucía}

Todo Sistema de Información se configura en una serie de procesos de entrada, tratamiento y difusión de la información. La creación del SIPHA implica la normalización de todos estos procesos documentales, así como de los sopor- tes de esa información, es decir equipos para el tratamiento y comunicación de la información.

Al respecto convendría hacer algunas precisiones sobre qué es la normalización y por qué es tan importante su aplicación a cualquier actividad. De acuerdo con Sanders, la normalización "es la forma de aplicar y establecer reglas con el fin de poner en orden un campo de actividad determinado, con el interés y concurso de todos los sectores afectados"7. Todo proceso de normalización implica la aplicación de reglas, sea cual sea el campo de actuación, con un objetivo fundamental: poner orden en una actividad. $Y$ esta "puesta en orden" posee unos componentes muy importantes de racionalidad, eficacia, economía..., elementos fundamentales en el desarrollo de cualquier actividad industrial, empresarial..., pero también documental. Es importante resaltar la necesidad de consenso de los sectores implicados a la hora de crear y aplicar una norma.

La normalización de equipos para el tratamiento y comunicación de la información y de técnicas y productos de información, con objeto de favorecer el intercambio y el acceso a la información tanto nacional como internacional, es un elemento fundamental de toda Política Nacional de Información. Y es éste también el objetivo del programa internacional UNISIST auspiciado por la UNESCO: contribuir al establecimiento de un Sistema Internacional de Información Científica y Técnica ${ }^{8}$. Luego, debe ser objetivo de todo Centro de Documentación e Información, como componente del Sistema Nacional de Información, contribuir a la compatibilidad e interconexión entre los diferentes sistemas de información; de ahí la importancia de conocer y aplicar, en la medida de lo posible, las normas que en relación con los procesos de entrada, tratamiento y salida de la información se generan por los organismos competentes (ISO, AENOR...). Éste es el hilo conductor que ha marcado las actuaciones desarrolladas por el Centro de Documentación.

Siempre se ha considerado prioritario la coordinación e integración entre las diferentes bases de datos del SIPHA y la posible interconexión con otros sistemas de información, de tal forma que cuando han existido normas, reglas aprobadas, se han intentado aplicar con las adaptaciones necesarias, y en el caso de no existir, se ha procurado conformarlas con el consenso de los sectores implicados. Conscientes siempre de que con la normalización de los procesos de entrada, tra-
I Ley 16/1985, de 25 de junio, del Patrimonio Histórico Español. BOE núm. I55 (29 de junio, 1985)

2 Plan General de Bienes Culturales. Sevilla 29 de Marzo de 1989. BOPA núm. 28I (18 de abril, 1989)

3 Decreto 107/1989, de 16 de mayo, por el que se crea el Instituto Andaluz de Patrimonio Histórico. BOJA, núm.46 (13 de junio, 1989)

4 López Yepes, José: Introducción al estudio de los sistemas de información y documentación en las organizaciones. En Fundamentos de Información y Documentación. Madrid: Eudema, 1989. Pág.204

5 Ibídem 2, pág.9353 envergadura y complejidad del SIPHA es suficientemente importante para plantearse como objetivo prioritario la creación de un Tesauro de Patrimonio Histórico, concebido en relación con el contexto en que se desarrolla y desde una interpretación polivalente del mismo SIPHA véase: Ladrón de Guevara, $M^{a}$ del Carmen: Experiencias del Centro de Documentación: El Sistema de Información del Patrimonio Histórico (SIPHA). En Catalogación del Patrimonio Histórico. Sevilla: Instituto Andaluz del Patrimonio Histórico, 1996, pp.58-71.

7 SANDERS, T.R.B.: Objectifs et Principes de la Normalisation, Pr. de Olle Sturen, Genève, International Organization for Standardization, p.19

8 UNESCO-UNISIST. Informe del estudio sobre la posibilidad de establecer un sistema mundial de información científica. París, 1970 
tamiento y salida de la información contribuiríamos a favorecer la calidad de la información y su eficaz recuperación, en resumen, a la eficacia del Sistema de Información. Evidentemente aún queda trabajo que desarrollar para la normalización del SIPHA.

Podemos establecer como objetivo fundamental del Sistema el posibilitar y favorecer el acceso a la información de los Bienes Culturales. Para hacer posible este objetivo fundamental se desarrollan una serie de procesos documentales.

- Procesos de entrada: consisten en la definición y captación de la información del SIPHA. Implica el establecimiento de criterios que determinen qué información debe formar parte del Sistema, la normalización de los circuitos documentales y el desarrollo de directrices para la elaboración de la documentación gráfica de los Bienes Culturales.

- Procesos de tratamiento: relacionados con la organización y el análisis de la documentación y la información para favorecer la recuperación eficaz de esa información por parte de los usuarios del Sistema. Se incluyen entre los procesos de tratamiento:

a) El diseño de las bases de datos, el establecimiento de los niveles de descripción y las normas de cumplimentación.

9 Ley $|/| 99 \mid$ de 3 de Julio de Patrimonio Histórico de Andalucía. BOJA, Año XIII, núm. 59 (I3, julio, 1991)

10 Ibídem 2, Preámbulo y art.2

II Martínez Montiel, Luis F. "La Base de Datos de Bienes Muebles del Patrimonio Histórico Andaluz". En Boletín Informativo, Año III, Número I0, Marzo 1994, pp.46-49.

12 Plata García, Fuensanta: Inventario y Catalogación del Patrimonio Etnológico de Andalucía. Antecedentes, Objeto y Proyectyo Inicial. En Catalogación del Patrimonio Histórico. Sevilla: IAPH, 1996, pp.86-93

Talego Vázquez, Félix: El Inventario de Arquitectura Popular de producción y transformación: el enfoque etnológico. En Catalogación del Patrimonio Histórico. Sevilla: IAPH, 1996, pp.94-107

13 Informe sobre el viaje realizado a Roma con motivo de la celebración de la "VIII Settimane per i Beni Culturali e Ambientali" (Roma, 7-13 Diciembre, 1992)

14 Decreto 19/1995, de 7 de febrero, por el que se aprueba el Reglamento de Protección y Fomento del Patrimonio Histórico de Andalucía. BOJA núm. 43 (17 de marzo, 1995) información que ha sido previamente seleccionada y tratada. Estos procesos están estrechamente relacionados con los anteriores; así el Tesauro de Patrimonio Histórico es un instrumento para el tratamiento y la recuperación de información. Se incluyen también entre estos procesos:

a) El establecimiento de niveles de acceso a la Información. Dependiendo del tipo de Patrimonio y del nivel de protección legal en el que se incluya, hay información a la que sólo tendrán acceso determinados usuarios del Sistema. Es por tanto importante establecer cuál es esa información y crear los filtros que sean necesarios en la consulta de las bases de datos.

b) El diseño de los módulos de consulta de las bases de datos de acuerdo con los niveles de acceso establecidos.

Por último es importante señalar que junto a la normalización de los procesos documentales ha de existir una normalización de los soportes de esa información: nos referimos a los equipos para el tratamiento y comunicación de la información. En el desarrollo del Sistema de Información se están siguiendo las recomendaciones y normas establecidas por el Servicio de Coordinación Informática de la Consejería de Gobernación.
Antes de centrarnos en la normalización de los procesos de tratamiento comentaremos brevemente la situación de los procesos de entrada de la información al Sistema.

El Sistema de Información se configura en dos grupos de bases de datos. El primer grupo incluye las bases de datos de información de los Bienes Culturales y el segundo las bases de datos de información bibliográfica, gráfica y documental de los Bienes Culturales y del Patrimonio Histórico en general.

Bases de Datos del Sistema de Información del Patrimonio Histórico(l).

- Base de datos de Bienes Inmuebles

- Base de datos de Patrimonio Arqueológico

- Catálogo de yacimientos Arqueológicos

- Registro de Excavaciones Arqueológicas(2)

- Base de datos de Bienes Muebles

- Inventario de Bienes Muebles de la Iglesia Católica

- Base de Datos de Museos

- Base de datos del Patrimonio Etnológico(3)

Base de datos del Patrimonio Documental y Bibliográfico(4)

Base de datos de las Fuentes de Información Bibliográfica

- Base de Datos de Información Bibliográfica (BIBLOS)

- Directorio de Bibliotecas

- Directorio de Revistas de Patrimonio Histórico

- Catálogo de Bases de Datos del Patrimonio Histórico.
- Base de datos de las Fuentes de Información Documental

- Guía de Fuentes

- Base de Datos de Información Documental

- Base de Datos Gráfica

- Base de datos de Actividades del Patrimonio

(1) Para más información véase:

Sistemas de Información sobre Patrimonio Histórico. I Jornadas sobre Catalogación del Patrimonio Histórico (Sevilla, 19-22 de abril de 1995).

Catálogo de Servicios del Instituto Andaluz del Patrimonio Histórico. 1996.

(2) Desarrollado por el Servicio de Investigación de la DGBC en colaboración con la Universidad de Granada.

(3) Desarrollada por el Servicio de Investigación de la DGBC.

(4) Por desarrollar.

El establecimiento de criterios para seleccionar la información que ha de incluirse en el Sistema es un proceso de normalización fundamental en el desarrollo de cada una de estas bases de datos. En el caso de las bases de datos de los Bienes Culturales (Bienes Muebles, Inmuebles, Patrimonio Arqueológico, Patrimonio Etnológico, Patrimonio Bibliográfico y Patrimonio Documental) es importante determinar qué bienes y actividades deben formar parte del Sistema. 
Es éste un aspecto directamente relacionado con la protección del Patrimonio Histórico. La Ley de Patrimonio Histórico Español y la Ley de Patrimonio Histórico de Andalucía establecen el nivel mínimo para que un bien sea protegido legalmente, y es su consideración como Patrimonio Histórico. Según la Ley de Patrimonio Histórico Español "integran el Patrimonio Histórico Español los inmuebles y objetos muebles de interés artístico, histórico, paleontológico, arqueológico, etnográfico, científico o técnico. También forman parte del mismo el patrimonio documental y bibliográfico, los yacimientos y zonas arqueológicas, así como los sitios naturales, jardines y parques, que tengan valor artístico, histórico o antropológico"I0.

Este concepto amplio de Patrimonio Histórico debe ser el punto de partida para la concreción de criterios que establezcan que un determinado objeto o actividad forma parte del Patrimonio Histórico y por tanto ha de incluirse en el Sistema de Información. Los responsables de las bases de datos de los Bienes Culturales trabajan en el desarrollo de estos criterios que deberán consensuarse con todas las partes implicadas, entre otras el Servicio de Protección de la DGBC. En este mismo Boletín se han presentado ya algunos criterios normalizados, en concreto referentes a la base de

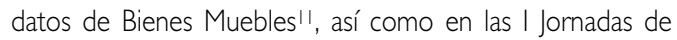
Catalogación del Patrimonio Histórico celebradas en abril de 199512. Somos conscientes de la importancia del establecimiento de criterios de selección de información para el desarrollo de un Sistema de Información de calidad sobre el Patrimonio Histórico de Andalucía.

En relación con las bases de datos documentales (Bibliográfica, Gráfica y Documental), se conciben fundamentalmente como instrumentos de información de los Bienes Culturales, de ahí que sean éstos el punto de referencia en el establecimiento de los criterios de selección e inclusión de información. Ahora bien, junto a ese criterio fundamental, en ocasiones también se selecciona información en función de una temática o línea de investigación general del Patrimonio Histórico.

La normalización de los circuitos de la documentación e información generada sobre los Bienes Culturales para su incorporación al Sistema de Información es otro aspecto importante de los procesos de entrada al Sistema, y para su desarrollo es fundamental la coordinación con todos los organismos e Instituciones de Patrimonio.

Desde el Centro también se trabaja en el desarrollo de criterios normalizados para la elaboración de la documentación gráfica de los Bienes Culturales, criterios que deberán tenerse en cuenta a la hora de incluir esta información en el SIPHA.

En relación con los procesos de tratamiento de la Información es importante determinar el nivel de descripción de las diferentes bases de datos con objeto de establecer una homogeneidad entre todas ellas. Desde nuestro punto de vista, el SIPHA es fruto de la integración de dos modelos de sistematización de la información de Patrimonio Histórico: el modelo italiano, que ha optado por la elaboración de un instrumento de descripción de un alto nivel de especificidad, que pretende recoger la máxima información sobre los Bienes Culturales; y el modelo francés, que ha optado por un instrumento de información básica que remite a la localización de la información existente en formato papel, donde se puede ampliar la información sobre el Bien'3.

El modelo que se está configurando podría considerarse un instrumento intermedio entre estos dos. En el diseño de las bases de datos se ha trabajado siempre desde un concepto de instrumento para el conocimiento; por tanto, se ha querido superar el nivel de descripción de inventario y desarrollar un modelo de instrumento que nos acercara al catálogo de conocimiento. De esta manera el SIPHA asume el concepto de Catálogo recogido en el Reglamento de Protección y Fomento del Patrimonio Histórico de Andalucía, entendido como instrumento administrativo y científico que posibilite la tutela jurídico-administrativa y que contribuya al conocimiento del Patrimonio Histórico y a la divulgación del mismo ${ }^{14}$.

En líneas generales puede decirse que, con las bases de datos desarrolladas hasta el momento, se ha cubierto una primera fase de información de los Bienes Culturales para la gestión. Ahora bien, el Sistema está concebido como un instrumento de conocimiento no sólo para la gestión sino también para las demás vertientes de la tutela del Patrimonio: investigación, conservación, difusión... En este sentido la informática ha puesto a nuestra disposición la posibilidad de configurar un instrumento flexible que permite incluir una información básica sobre el bien, pero que posibilita la ampliación y cualificación de esa información de manera que el Sistema pueda cumplir su objetivo fundamental de servicio de información para la tutela integral del Patrimonio Histórico.

En relación con la normalización de los formatos de las bases de datos es importante la definición de los bloques de información y la organización y distribución de los mismos en la estructura general de la base de datos. A este respecto, en la actualidad se trabaja en la definición de las tablas comunes de las diferentes bases de datos para proceder a su integración.

En el diseño de las bases de datos de los Bienes Culturales (Bienes Muebles, Inmuebles, Patrimonio Arqueológico...), se han estudiado diferentes modelos de fichas, tales como las del Instituto Centrale per il Catálogo e la Documentazione de Roma, Bases de Datos del Ministerio de Cultura Francés, Carta Arqueológica de Aragón, Base de Datos Arcat de Arte Románico catalán (Centre D'Art Románic Catalá), Base de Datos de Yacimientos Arqueológicos de la Comunidad de Madrid, etc. Asimismo, en el diseño de las bases de datos documentales (bibliográfica, gráfica y documental) se han análizado normas internacionales, tales como las Reglas de Catalogación para monografías y materiales especiales del Ministerio de Cultura, la Norma Internacional General de Descripción Archivística (ISAD (G)) aprobadas por el Consejo Internacional de Archivos (CIA), etc. También se han elaborado unas normas de cumplimentación de todas las bases de datos diseñadas hasta el momento.

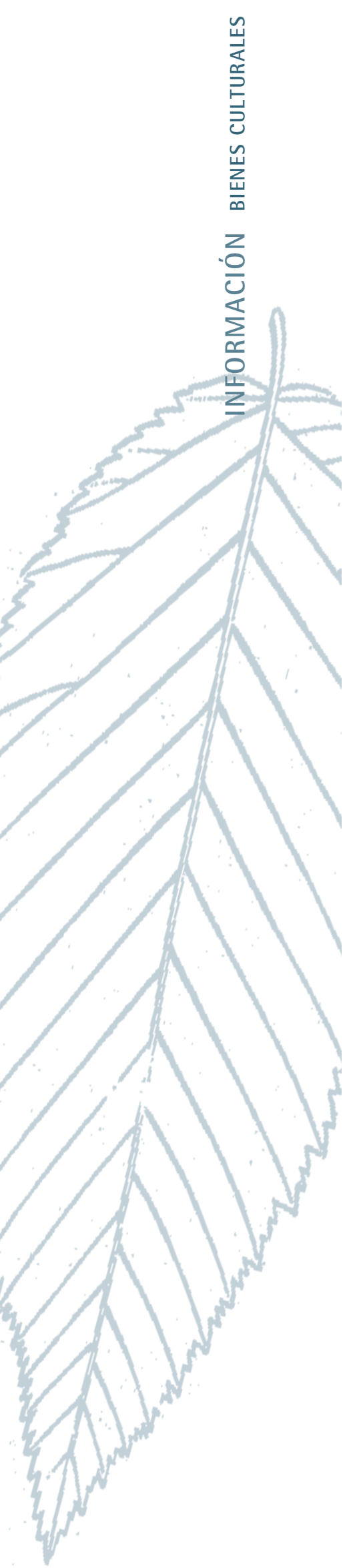




\section{EI Tesauro de Patrimonio Histórico}

En cuanto a la normalización terminológica, la envergadura y complejidad del Sistema era lo suficientemente importante como para que se planteara como objetivo prioritario el desarrollo de un Tesauro de Patrimonio Histórico concebido como lenguaje documental del Sistema de Información que permita la integración de las bases de datos y facilite de forma efectiva la recuperación de la información.

El Tesauro de Patrimonio Histórico que se desarrolla en colaboración con la Universidad y las Instituciones de Patrimonio es un proyecto ambicioso y complejo. Hay una serie de elementos que sin duda lo condicionan y caracterizan, y que conviene analizar para comprender las necesidades que esta herramienta de trabajo debe cubrir en el desarrollo del Sistema de Información.

En primer lugar la Ley de Patrimonio Histórico Español, como ya hemos comentado, expone un concepto muy amplio de Patrimonio Histórico. Son varias las disciplinas que conforman ese concepto: Arqueología, Etnología, Arte y Arquitectura..., y el lenguaje documental del Sistema debía ser útil a todas ellas.

En segundo lugar el Tesauro de Patrimonio Histórico debía permitir la interrelación y la recuperación de información no sólo sobre objetos (muebles, inmuebles, ...) y actividades, sino también sobre documentación (bibliográfica, gráfica y textual). Ésta era otra condición que debía tenerse presente en su desarrollo, pues evidentemente no son los mismos descriptores los que se utilizan cuando analizamos un objeto que cuando se trata de un libro, o un documento textual o gráfico: el tipo de descriptor, el nivel de especificidad... varía de forma notable.

A partir de estos condicionantes la cuestión fundamental que debía solucionarse era si se elaboraría un Tesauro de Patrimonio Histórico o un Tesauro para cada una de las disciplinas que integran el Patrimonio Histórico: Arqueología, Etnología y Arte y Arquitectura... Para responder a esta cuestión analizaremos brevemente las actuaciones que fueron desarrollandose y ayudaron a resolverla.

En una primera fase se procuró conocer la existencia de experiencias similares desarrolladas en España y fuera de nuestras fronteras, pues siempre hemos sido conscientes del coste de este tipo de proyectos y de la necesidad por tanto de no repetir esfuerzos. Así fue como conocimos experiencias como la desarrollada por la Sección de Museos de la Generalitat de Cataluña en relación con el programa DAC, Programa de Documentación Asistida de Colecciones, que está siendo llevado a cabo, conjuntamente por esa Sección y el Ayuntamiento de Barcelona. En este proyecto existen ochenta Diccionarios ${ }^{15}$

Por otro lado, en lo que respecta a los Museos de Titularidad Estatal, la Dirección de los Museos Estatales de la Dirección General de Bellas Artes y Archivos del Ministerio de Cultura, a través de una Comisión de Documentación está desarrollando un proyecto de documentación en museos. Dicha Comisión está elaborando vocabularios de diferentes materias con objeto de que en un futuro puedan desarrollarse tesauros ${ }^{16}$.

Se contactó también con el Centro de Información y Documentación del CSIC (CINDOC) para conocer los tesauros existentes relativos a Patrimonio Histórico. Se analizaron algunos de ellos tales como: el Tesauro de Arte y Arquitectura de la Fundación Paul Getty (AAT), el Tesauro Iconográfico de F. Garnier (I98I), el Tesauro Arqueológico de la Comisión Real de Monumentos Históricos de Inglaterra (1986), El Sistema de Clasificación de Documentación Etnográfica Española (1993), entre otros. Ninguno de ellos servía íntegramente para el desarrollo del Sistema. Sin embargo, se observó que de todos ellos, así como de otros que se han utilizado posteriormente, se podía extraer información útil para el desarrollo del lenguaje documental.

Nuestra primera intención fue cubrir las necesidades de cada una de las disciplinas que forman parte del Patrimonio Histórico, elaborando un lenguaje documental para cada una de ellas. En relación con Etnología se contaba con el Sistema de Clasificación de Documentación Etnográfica Española, respecto a Arte y Arquitectura existía el Tesauro de Arte y Arquitectura de la Fundación Paul Getty. Y fue precisamente del análisis de este tesauro de donde extrajimos conclusiones que serían definitivas para el desarrollo del Tesauro. Casi inmediatamente se observó que la estructura por facetas que presentaba era lo suficientemente flexible como para permitir una posible integración de las diferentes disciplinas de Patrimonio Histórico en un único Tesauro. Aunque se pensó en un primer momento la posibilidad de traducir el tesauro, pronto comprendimos que la traducción sólo habría resuelto en parte nuestras necesidades: en primer lugar porque el tesauro era sólo de Arte y Arquitectura y quedaba aún la especialidad de Arqueología sin cubrir; $y$, por otro lado, porque incluso para la disciplina de Arte y Arquitectura, la realidad del Patrimonio artístico andaluz y español era sustancialmente diferente a la realidad recogida en el AAT, lo que implicaba adaptaciones sustanciales en el Tesauro de la Fundación.

Se adaptó la estructura por facetas del AAT y se comenzó a trabajar en el desarrollo de un primer borrador, al hilo de las necesidades surgidas en el área de Bienes Muebles, en concreto en el desarrollo del Inventario de Bienes Muebles de la Iglesia. Este borrador fue el punto de partida para extraer una serie de conclusiones que marcarían el desarrollo del Tesauro.

Conforme se trabajaba en la recopilación de términos de Arte y Arquitectura y en el desarrollo de las primeras facetas (Estilos y Periodos Culturales, Materiales, Técnicas, Tipologías, Mitología e Iconografía...) percibimos que había algunas que podían ser comunes a las distintas disciplinas. Había bastantes elementos comunes como para que se concibiera la posibilidad de desarrollar un lenguaje documental que permitiera la integración de todas ellas, aunque respetando las especificidades de cada una. Efectivamente, esta primera experiencia nos permitió tomar conciencia de que realmente tenía sentido plantear el desarrollo de un único instrumento: un Tesauro de Patrimonio Histórico que fuera útil a todas las bases de datos que se estaban configurando. 
Existen razones prácticas que justifican el desarrollo de un único tesauro para el SIPHA, razones que resultaron evidentes cuando se formó el grupo de trabajo para su desarrollo, como es la cantidad de términos comunes entre las diferentes disciplinas, lo que hacía inviable el desarrollo de un tesauro para cada disciplina. Pero quizás la razón más importante es que el Patrimonio Histórico no está formado por compartimentos estancos independientes sin ningún tipo de relación, sino que por el contrario el Patrimonio ha de concebirse en relación con el contexto en que se desarrolla, como ya hemos apuntado anteriormente, y desde una interpretación polivalente del mismo. Así pues ese concepto integrador del conocimiento e interpretación del Patrimonio Hitórico que recoge el Sistema de Información, debía hacerlo suyo el lenguaje documental de ese Sistema. En este sentido, un instrumento que permita la posibilidad de acercarse al Patrimonio desde diferentes enfoques y posibilite la descripción de un mismo objeto desde diferentes perspectivas (artística, antropológica y arqueológica), nos parecía sin duda ambicioso y novedoso.

Fuimos conscientes de las dificultades que este proyecto entrañaba en sí mismo, por razones bastante obvias. Los intentos de integración del lenguaje documental de diferentes disciplinas pueden generar recelos por razones diversas que no vamos a analizar aquí. Por otro lado, también es cierto que ese esfuerzo de integración implica la realización de concesiones por parte de cada disciplina, pero estamos convencidos de que son muchas más las ventajas que los inconvenientes del proceso de integración. Por último, no es ésta la primera ni la única experiencia de tesauros o macrotesauros multidisciplinares. Son numerosas las experiencias en este sentido, tales como el Tesauro de la UNESCO entre otros.

En cualquier caso creemos que el intento de integración y acercamiento entre diferentes disciplinas con puntos en común es importante por cuanto supone un enriquecimiento que sin duda debe significar un avance.

A pesar de todas estas reflexiones internas, creímos conveniente realizar un debate en el que estuvieran presentes todos los futuros usuarios del Tesauro de Patrimonio Histórico, con objeto de concretar el tipo de tesauro que demandaba el Sistema de Información del Patrimonio Histórico. Así fue como se organizó un Seminario especializado en metodología de construccción de Tesauros en Junio de 1995, que fue impartido por Don Antonio García Gutiérrez, Catedrático de Documentación de la Facultad de Ciencias de la Información de la Universidad de Sevilla. En el seminario estuvieron presentes representantes de la Universidad y de las Instituciones de Patrimonio Histórico, con objeto de que expusieran sus puntos de vista.

El tema central del debate fue el tipo de tesauro a realizar. Se discutieron dos posibilidades: un macrotesauro del Patrimonio Histórico que abarcara todas las especialidades, o bien realizar varios tesauros paralelos por cada especialidad. Hubo opiniones a favor y en contra de ambas opciones. En resumen las conclusiones del debate fueron:

- Se acordó la elaboración de estructuras independientes por disciplinas, para posteriormente intentar determinar los elementos comunes de las tres especialidades.

- El Tesauro debía ser un instrumento útil para el análisis de objetos y de documentación e información bibliográfica, gráfica y textual.

Una vez concluido el seminario se formó un grupo de trabajo para el desarrollo del Tesauro integrado por representantes del IAPH, la Universidad y las Instituciones de Patrimonio Histórico. Fueron necesarias pocas reuniones para que los integrantes del grupo de trabajo llegaran a las mismas conclusiones que habínn llegado los documentalistas del Centro de Documentación: había razones cuando menos prácticas que evidenciaban la necesidad de elaborar un único Tesauro de Patrimonio Histórico estructurado por facetas.

El grupo de trabajo está siguiendo la metodología recogida en la Norma ISO 2788-1986 (E) de "Directrices para el establecimiento y desarrollo de tesauros monolingües". Hasta el momento han concluido la macroestructura del Tesauro que fue presentada el 19 de Enero a la Comisión de Seguimiento del Tesauro que está integrada asimismo por representantes de las Instituciones de Patrimonio y de la Universidad.

La macroestructura del Tesauro recoge diferentes facetas, tales como: Patrimonio Histórico, Culturas Históricas, Estilos Artísticos, Periodos Geológicos, Periodos Históricos, Agentes, Materiales, Actividades, Objetos, Estructuras, Disciplinas,... y una serie de listados auxiliares en los que se incluyen los Bienes Culturales codificados, Iconografía y Mitología, Términos geográficos de Andalucía,...

Está previsto que en el verano de 1996 se presente lá primera versión del Tesauro de Patrimonio Histórico. En sư momento lo haremos público con objeto de que el trabajo realizado por el IAPH pueda ser útil a cuantos tengan necesidades similares en el conocimiento y sistematización de la documentación e información de Património Históriço, con. el convencimiento de que en el ámbito de la Documentación como en cualquier otro sector de actividad hay que evitar la repetición de proyectos costosos en tiempo y en recursos

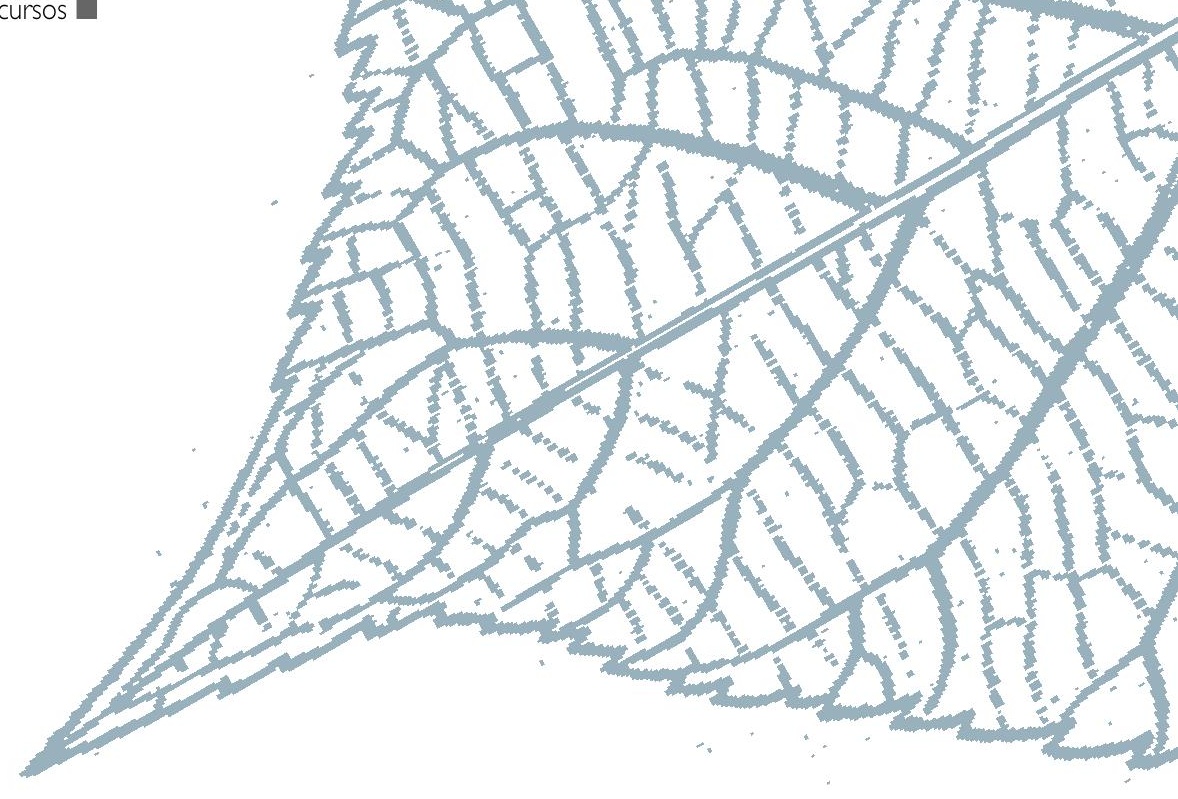

